\title{
A Conceptual Overview of Energy Consumption based Routing protocol - ECBR
}

\author{
Ashish Srivastava \\ BBD University (CSE) \\ Lucknow India
}

\author{
Atul Mishra \\ BBD University (CSE) \\ Lucknow India
}

\author{
Shraddha Upadhyay \\ BBDNITM (CSE) \\ Lucknow India
}

\begin{abstract}
Working on limited energy within an Ad-hoc network requires a strong communication channel. In this paper, we are trying to propose a new routing protocol which is based on Energy consumption for mobile ad-hoc networks. Since MANET is an infrastructure less network, so the nodes form the network from which they do the communication and message passing. Each nodes itself act as router. Energy is consumed in sending or receiving the packets between the nodes. Here we are measuring the Energy consumption in different states and adding a new Energy consumption field in Routing Table as well. Energy consumption based Routing (ECBR) protocol is thus a new benchmark in which the routing will consider the energy consumption for selecting the path. Doing so will reduce the chances of network failure. We show that mechanism is stable, counter-active, reliable and fair.
\end{abstract}

\section{Keywords}

ECBR, QoS, MANET, Routing.

\section{INTRODUCTION}

In MANET [15], each node is dynamic in nature and can join or leave network according to the use resulting in the change of topology as well. The nodes in the network are normally power curb because of their dependency on limited power. In wireless communication, especially in MANET, remarkable amount of energy is consumed not only in transmission among the nodes but also in overhearing of the packets send from other nodes. The one of the most important objectives of MANET routing protocol is to maximize energy efficiency, since nodes in MANET depend on limited energy resources. The possible energy consumption states are: transmit, receive, idle and sleep. An energy consumption model in terms of the costs of both sending and receiving traffic is presented in Feeney et al [8].

The main objectives of MANET routing protocols are to maximize throughput (network), energy efficiency, network lifetime and to minimize the delay. The network throughput is usually measured by packet delivery ratio while the most significant contribution to energy Consumption is measured by routing overhead which is the number or size of routing control packets [9].

Ad-hoc networks are peer-to-peer multi-hop wireless networks where the packets are transmitted in a stored and forward manner from a source to an arbitrary destination. There networks are self-configure, self maintain [1] and selfcontrolled networks. These networks sometimes also called as "Infrastructure less network". The Ad-hoc networks don't have any centralized Access point or Base point for
Communication, but it works by communicating through wireless links both, in the packet forwarding and route discovery. The dynamic nature of the nodes in MANET results continuous change in the topology as well. Hence, each nodes work as a Router viz. the active nodes in the network act as both the participant and routers for routing and message passing.

The "openness" of the network architecture and its dynamic network topology imposes restrictions on MANET [2]. In MANET, all devices have an equal status and are free to communicate with one another within a circle. Self organizing behavior of mobile nodes makes deployment in any environment viz. in shopping mall, theaters or even in streets. Even these types of networks can be used in disasters, relief, rescue, education, monitoring system, excavation, intelligent transport system, battle fields etc.

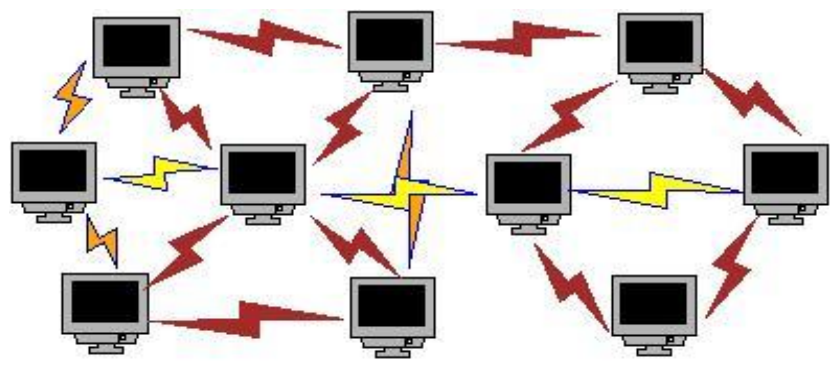

Fig 1 : Multi hopping in Ad-hoc Network

The rest of the paper is organized as follows. The next section we discuss routing in Mobile Ad-Hoc network and its types. Also, we propose a new MANET routing protocol based on energy consumption model. In section we present the previous work done in the field of MANET related to routing protocols and energy consumption. In the section 4 we exhibit the new approaches to evaluate energy efficiency in MANET routing protocol and details of application of energy efficiency metrics to the protocols. In section 4 explain how we generate our scenarios. Section 5 will present the conclusion and future work.

\section{ROUTING OVERVIEW IN MANET}

Mobile ad hoc network (MANET) typically refers to any set of networks where all devices have equal status on a network and are free to associate with any other ad hoc network device in its association range. Ad hoc network usually quote to a mode of proceeding of IEEE 802.11 wireless networks.

Routing in MANET is quite different from traditional routing found on Infrastructure networks. Routing protocols in MANET depends on many factors viz. topology, router selection, initiation of request and specific underlying 
characteristics. Highly dynamic nature of these networks imposes severe restrictions on routing protocols. Also the low resource availability of these networks demands efficient utilization and the motivation for optimal routing in Ad-hoc networks.

One of the striking challenge in designing a routing protocol for Ad-hoc networks depends on the fact that, on one hand, a node needs to know the route information for determining a route for transmission and, on the other hand, the topology keeps on changing due to the mobile environment. The situation become more challenging, as the number of network nodes increase, finding the route to the destinations also requires large and frequent exchange of routing control information among the nodes.

\section{MANET ROUTING PROTOCOL}
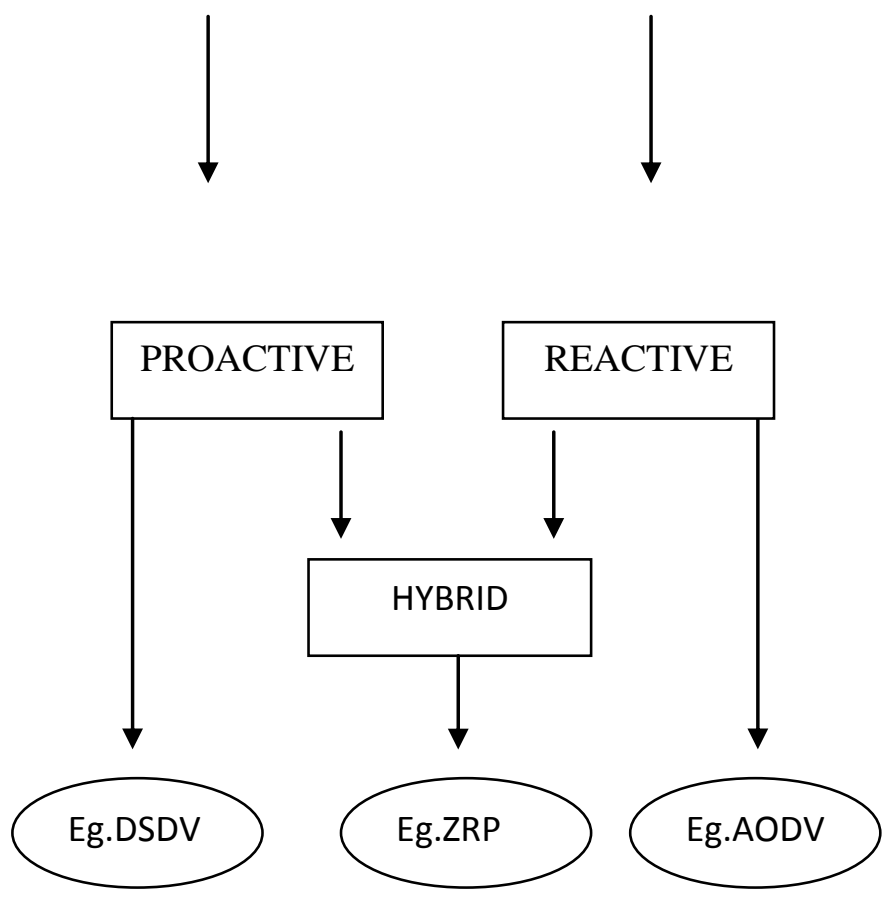

Fig 2 : Types of Routing

Ad hoc routing protocols are broadly classified as: Proactive (or table driven) and Reactive (on demand). A proactive protocol states that nodes in a Mobile Ad hoc network should keep the record of route to all probable destinations so that route can be indentified when needed. Reactive protocols, on the other hand, employs lazy approach where nodes only discover routes to destination on demand.

\section{RELATED WORK}

In order to achieve Quality of Service (QoS), several routing models have been proposed, Vinh Dien et. al. [14] proposed an efficient load balancing approach. According to the author, the source node periodically sends probe packets on all paths to the destination and waits for these packets to be sent back by the destination. By measuring the information in the probe packet such as the round trip time, the number of congested packet, etc, the source node could estimate on which path it should send more data, and on which path it should send less.
The on-demand routing is famous and important in the MANET [4]. Instead of exchanging route messages to maintain a permanent route table, the on-demand routing protocols assemble routes only when a node is required to send the data to a destination. The base protocols of the on demand protocols are the Dynamic Source Routing (DSR) [12] and the Ad hoc On-demand Distance Vector (AODV) [13] routing. However, these protocols do not support multipath. Multiple paths can be useful in improving the effective bandwidth of communication pairs, responding to congestion and traffic, and increasing the reliability.

Xuechao et.al. [5] Examine the effect on the distribution of input traffic among multiple paths in AODV-MP (Ad Hoc onDemand Distance Vector Routing with Multi-Path). It was examined by giving the essence of the AODV-MP, and then designing and realizing a load-balancing algorithm based on AODV-MP made up of independent nodes, in which source node distributes packets reasonably in the several paths according to the load state of the network.

PP \& SP [3], extend the Dynamic Source Routing (DSR) protocol with the load balancing function. When the Source node wants to send packets to the destination node; it will use the DSR protocol to find all possible paths to the destination. It will also measure the number of congested packet on each path based on the information sent by the destination node in the Route Reply packets. The source node then distributes the data packets on all paths in the way that the total number of congested packets on each path is equal. From time to time the destination node will update the number of congested packet by sending the Load Packet (LP) to the source node and the load balancing decision in the source node is made accordingly with this updated information.

\section{ECBR : ENERGY CONSUMPTION BASED ROUTING}

\subsection{Statement}

In MANET, routing is difficult due its dynamic nature. Multihopping networks use the shortest path for routing which is not adequate, nodes in the network acts as both an end system and router. Hence, an additional energy is required in transmission.

In proposed Energy consumption based routing model, addition of a new field of Energy Consumption is proposed. Now the routing table will include the calculated energy and this parameter will decide the new route. The aim is to evaluate the availability of energy resources and use them efficiently to gain maximum output. This will work whenever a node sends a packet to another node in the network. Energy consumption parameter will be evaluated and calculated (refer to next section). This value will then be stored and updated by the nodes. In ECBR, thus, the route discovery and selection will be done not only on the basis of shortest path but also on the amount of Energy consumed during that process and Source node can select the best from the all. Figure 5 represent the flow chart of overall scenario.

\begin{tabular}{|c|c|c|c|c|l|l|l|}
\hline Mask Network & $\begin{array}{c}\text { Next } \\
\text { hop } \\
\text { Address }\end{array}$ & Anterface Flags & $\begin{array}{l}\text { Reference } \\
\text { Count }\end{array}$ & Use & $\begin{array}{l}\text { Energy } \\
\text { Consum } \\
\text { ption }\end{array}$ \\
\hline
\end{tabular}

Fig 3 : Proposed packet format 


\subsection{Energy Calculation}

Information of Energy consumption will surely prove to be an important aspect in routing and further can be used in QoS route discovery. The calculation of Energy consumption can be done in four states/modes viz. Transmit, Receive, Idle and Sleep.

- Transmission Mode [11]: In transmission mode a node communicates data packet to other nodes present in the network. For transmitting the data packets these nodes need Energy which is called Transmission Energy (TMx). The amount of Energy spent during transmitting is calculated by:-

$$
\begin{aligned}
& \mathrm{TM}=(330 * \text { Packet size }) / 2 * 10^{6} \\
& \mathrm{TP}_{\mathrm{R}}=\mathrm{TM} / \mathrm{T}_{\mathrm{t}} \quad \text { (where, } \mathrm{TP}_{\mathrm{R}} \text { is Transmission Power) } \\
& \text { (Where, Packet size is specified in bits) }
\end{aligned}
$$

- Reception Mode [11]: When a node receives a data packet from other node in a network then it is called Reception mode and Energy taken to receive that packet is called Reception Energy (RMx). It is calculated as:

$$
\begin{aligned}
& \mathrm{RM}=(230 * \text { Packet size }) / 2 * 10^{6} \\
& \mathrm{RP}_{\mathrm{R}}=\mathrm{RM} / \mathrm{T}_{\mathrm{r}} \quad \text { (where, } \mathrm{RP}_{\mathrm{R}} \text { is Reception Power) }
\end{aligned}
$$

( $T_{r}$ is the time taken to receive data packet.)

- Idle Mode [11]: In this mode node is neither transmitting nor receiving any data. But is waiting for any packet to transfer, because of this nodes consumes power since it communicates in wireless medium continuously. The power consumed in Idle Mode is

$\mathrm{PI}=\mathrm{PR}$ where, PI is power consumed in
Idle Mode and PR is power consumed in Reception
Mode.

- Sleep Mode: It is a low power state where the node can neither receive nor transmit.

\subsection{Design And Technical Constraints}

Security: Controlling access to network resources is a primary concern. Routing protocols provide techniques that can be used as part of a security strategy. It is also advised to insert a filter on the routes being advertised so that certain routes are not advertised in some parts of the network. In this paper, we are not applying any filter strategies but future work will include so.

Convergence: When network topology reorganizes, network traffic must transform quickly. The terminology "convergence time" exemplify the time it takes a router to start using a new route after a topology changes.

The moment topology changes router must perform three tasks:

- $\quad$ Detect the change

- Select a new route

- Circulate the transformed route details.

Route Selection: Energy Consumption based Routing Protocol (ECBR) (refer Fig. 4) compare route metrics to select the best route from a group of possible routes. Route metrics are enumerated by allotting a characteristic or set of characteristics to each physical network. The concern for the route is a collection of the characteristics of each physical network in the route.

Scalability: The ability to extend your internetwork is adamant, in chunk, by the soaring characteristics of the routing protocols used and the quality of the network design. Network scalability is limited by two factors: operational issues and technical issues .Generally, operational issues are more apparent than technical issues. Operational scaling covers the use of large areas or protocols that does not demand hierarchical structures. When hierarchical protocols are prescribed, technical scaling encourages the use of small areas.

Route Summarization: In Energy Consumption based Routing Protocol (ECBR), routers can deduct some sets of routes to a specific advertisement, chopping both the burden on the router and the observed complexity of the network. The importance of route summarization kept rising with network size.

Memory: Routing protocols uses stockpile to save routing tables and topology information. Route summarization trims memory consumption for all routing protocols. Small areas minimize the memory consumption for hierarchical routing protocols.

CPU Requirements: CPU manipulation is protocol dependent. Even some protocols use CPU cycles to compute new routes to existing routes. Other protocols use CPU cycles to recreate routing tables after the transformation of topology.

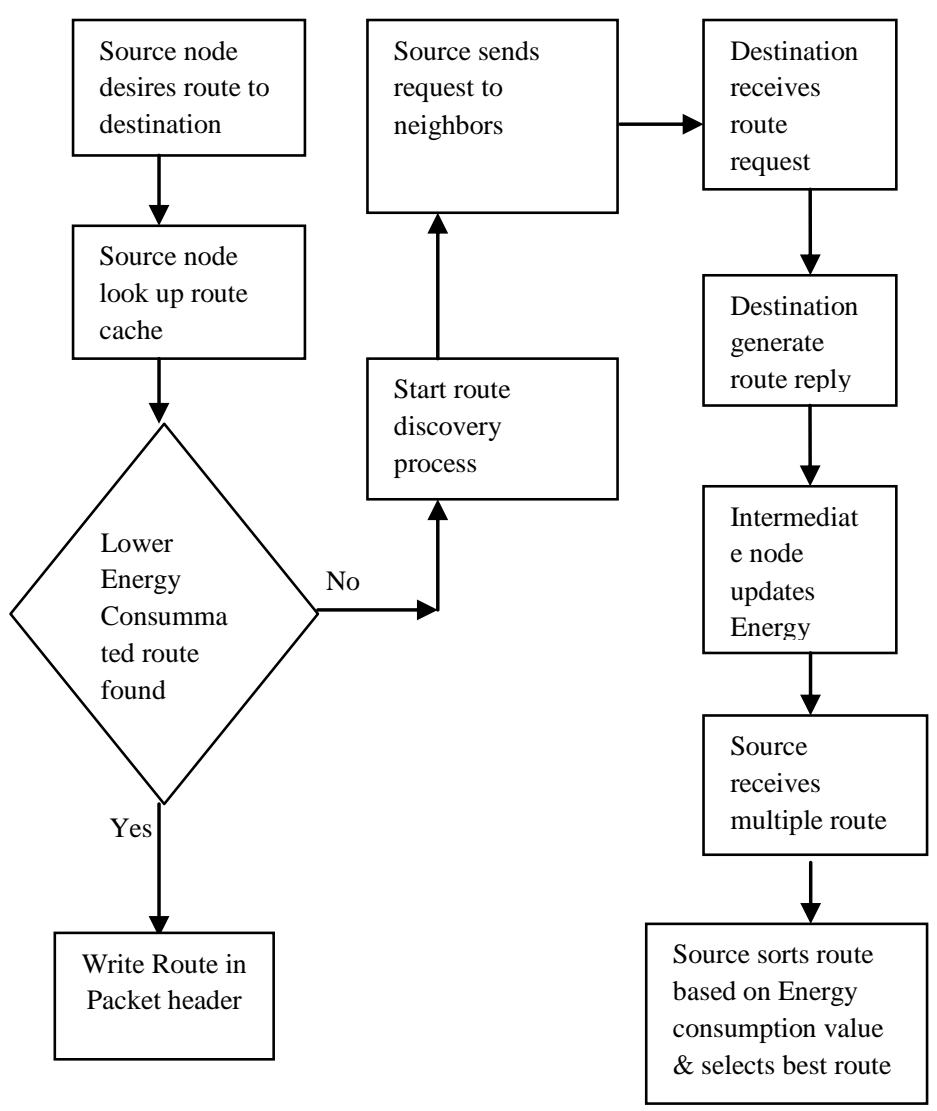

Fig 4 : Flow chart of the Route Discovery 
Bandwidth Requirements: The use of Bandwidth is also dependent on protocol. Three vital concerns determine the size of bandwidth a routing protocol consumes:

- When to send the routing information - After regular intervals Periodic updates is to be sent. Flash updates are sent only when a transition occurs.

- What type of routing information is to send - Routing information of the complete updates. Incomplete updates contain only changed information.

- Where to send routing information - Flooded updates is to be send to all the routers. If a router is affected by a change then bounded updates is to be send to it only.

\section{RESULT ANALYSIS}

Based on the result obtained we can conclude the effect of packet size on corresponding energy consumption. The aim is to reduce the difference in energy consumption between the nodes. This may lead to longer network life and longer time to node failure.

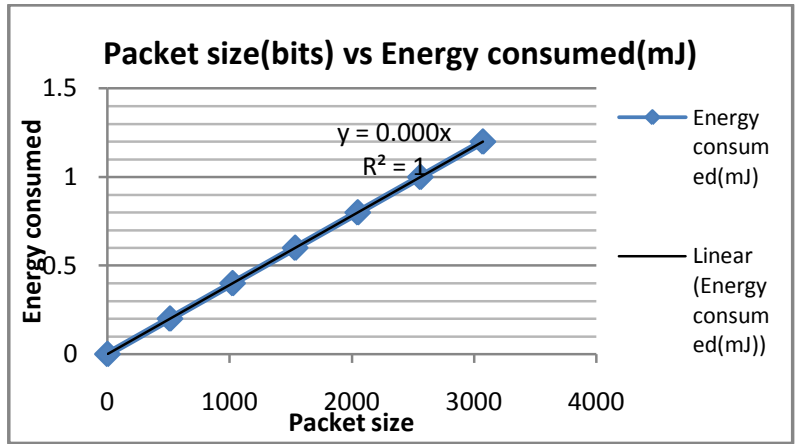

Fig 5 : Packet Size vs. Energy Consumption

Energy consumption is directly proportional to the relative packet size. Fig 5 represents a linear graph showing that energy consumption increases with increase in size of packet.

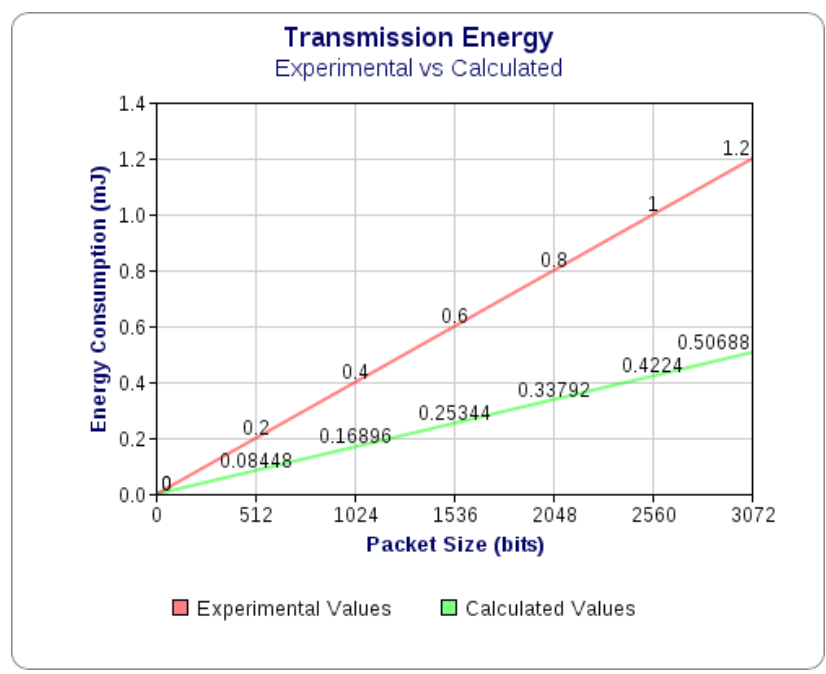

Fig 6 : Transmission Energy
Figure 6 and 7 represent the Transmission energy and reception energy respectively. It is clear from the graph illustrated (Fig $6 \& 7$ ) that energy increases with increase in packet size. Validity of these metrics is verified and they clearly demonstrated that significant reductions in cost can be obtained by using shortest-path routing. It is also observed that energy consumed is directly proportional to the Packet size.

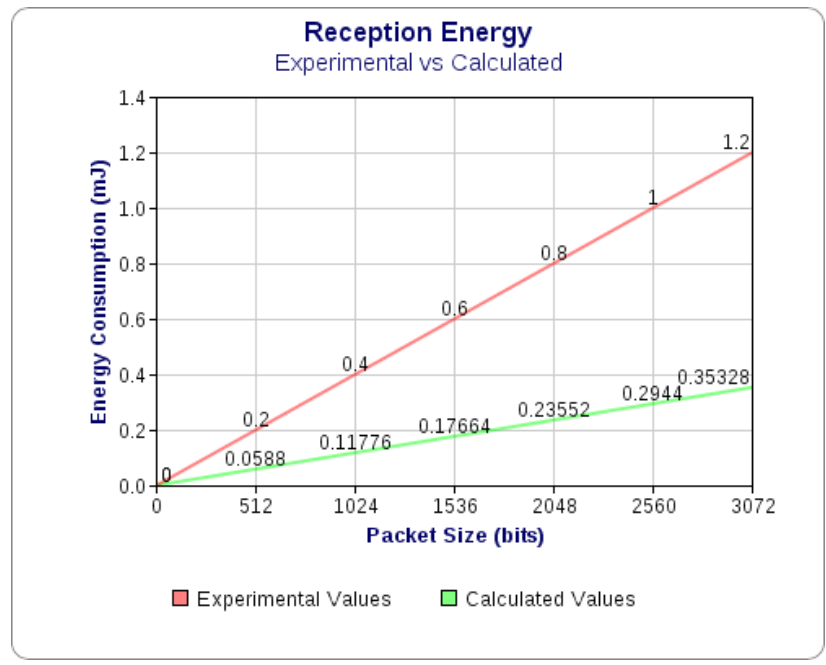

Fig 7 : Reception Energy

\section{CONCLUSION}

On the basis of above discussion, we can conclude that using Energy consumption metrics to discover routes is very beneficial. The specific conclusions from this conceptual overview are:

- Large scale networks have excessive cost decrement.

- Energy consumption is directly proportional to the size of packet. This means that energy consumption will increase with the increase in the packet size.

- Energy saving will be more in Denser networks.

- Energy saving are negligible at very High loads and works efficiently at moderate network loads.

\section{FUTURE WORK}

In this paper, the need to make routing protocols based on Energy Consumption. Thus, rather than using traditional metrics such as reference count or transmission delay for finding routes, we suggest that it is more important to use node cost (which are functions of remaining battery power) as metrics A feature of our metrics is that they can be easily incorporated for use in existing routing protocols for ad hoc networks.

Future work will include the implementation of ECBR in real network scenario. It is worth pointing out that our results will hold true in networks where nodes are mobile. This is because nodes in real networks do not move randomly independently. Rather, clusters of nodes move in correlated ways. 


\section{REFERENCES}

[1] W.Lou, Y.Fang, "A survey on wireless security in mobile ad hoc networks: challenges and available solutions," Book chapter in Ad Hoc Wireless Networking, Kluwer, pp.68-73, May 2003.

[2] Salaheddin Darwish, Simon J.E.Taylor and Gheorghita Ghinea, "Security Server-Based Architecture for Mobile Ad hoc Networks," IEEE $11^{\text {th }}$ International Conference on Trust, Security and Privacy in Computing and Communications, 978-0-7695-4745-9, 2012

[3] P. Pham, S. Perreau, "Multi-path routing protocol with load balancing policy in mobile ad hoc network", 4th International Workshop on Mobile and Wireless Communications Network, Sept 2002, pp. 48 - 52.

[4] C.Hieu, D.Kim, C.Hong and Y.Bang," On-demand Multi-path Balancing in Wireless Mesh Networks". International Conference on Information Networking, 2009, ICOIN Issue Date: 21-24.

[5] I.Xuechao, X.Chunxiu, W.Muqing, Z.Yan and W.Dapeng, "Design and Realization of a Novel Multipath Load-Balancing Routing Protocol in Ad Hoc Network", in Proceedings of IEEE WASE International Conference on Information Engineering, 2009, pp. 247250

[6] N.Wisitpongphan, G. Ferrari, S. Panichpapiboon, J.S. Parikh, and O.K. Tonguz, "QoS provisioning using BERbased routing for ad hoc wireless networks “,IEEE Vehicular Tech. Conf.(VTC Spring 2005), PP.24832487.

[7] G.IIanchezhiapandian, P.Sheik Abdul Khader, "Quality of Service (QoS) Routing in Mobile Ad-hoc Network (MANET) using AODV protocol: Cross-Layer Approach", International Journal of Computer Applications (0975-8887) $2^{\text {nd }}$ National Conference on Future Computing February 2013.
[8] LAURA MARIE FEENEY ,"An Energy Consumption Model for Performance Analysis of Routing Protocols for Mobile Ad Hoc Networks", Mobile Networks and Applications, Volume 6,Issue 3,June 2001,pages 239-24

[9] L.M.feeney, M. Nilsson, "Investigating the energy consumption of a wireless network interface in ad hoc networking environment," in Proceedings of IEEE Infocom, April 2001

[10] A.K.Vatsa, Prince Chauhan, Meenakshi Chauhan and Jyothi Sharma, "Routing Mechanism for MANET in Disaster Area", International Journal of Networking and Mobile Technologies, Vol 2/ISSUE 2/MAY 2011.

[11] Dharam Vir, Dr. S.K. Agarwal, Dr.S.A. Imam, “ A Simulation Study on Node Energy Constraints of Routing Protocols of Mobile Ad hoc Networks use of QualNet Simulator", International Journal of Advanced Research In Electrical, Electronics and Instrumentation Engineering Vol.1,Issue ,November 2012.

[12] J.Broch, D.Johnson and D.Maltz "Dynamic Source Routing Protocol for Mobile Ad Hoc Networks", IETF Internet draft, 2004.

[13] Belding-Royer, Elizabeth M., and Charles E. Perkins. "Evolution and future directions of the ad hoc on-demand distance-vector routing protocol." Ad Hoc Networks 1.1 (2003): $125-150$

[14] Vinh Dien Hoang, Shao, Z. ; Fujise, M. "Efficient Load Balancing in MANETS to Improve Network Performance", ITS Telecommunications Proceedings, 2006 6th International Conference on 2006

[15] Goyal, Priyanka, Vinti Parmar, and Rahul Rishi. "Manet: vulnerabilities, challenges, attacks, application." IJCEM International Journal of Computational Engineering \& Management 11 (2011): 32-37. 